\title{
Epiphora: a quick fix?
}

\section{Abstract}

Purpose To assess whether any simple, easily performed and minimally invasive procedure may help the symptoms of patients with functional nasolacrimal obstruction without recourse to elaborate investigation or major surgery. A randomised study was performed to assess the success of retropunctal cautery (RPC) and one-snip punctoplasty in this condition.

Methods In the absence of any cause for excess lacrimation, eyelid malposition or nasolacrimal obstruction, patients underwent either syringing alone (group $A, n=15$ ) or syringing with RPC and a one-snip punctoplasty (group $B, n=15$ ).

Results Three months after treatment, six patients in group A were improved, compared with 13 in group B (Fisher's exact test, $p=0.0096$ ).

Conclusions We would recommend that patients who suffer from a pump or functional nasolacrimal obstruction should receive RPC and a one-snip punctoplasty following the demonstration of a patent system on syringing. These simple procedures would not impair further investigation or lacrimal surgery if required in the future.

Key words Epiphora, One-snip punctoplasty, Retropunctal cautery, Syringe

Epiphora is a common symptom in both the young and the elderly. It may be functionally disabling, as well as cosmetically embarrassing. Causes include excess lacrimation, eyelid malposition, nasolacrimal obstruction, or a combination of these. The surgical management in these cases is well known, ${ }^{1}$ but epiphora in the absence of these factors, presumed to be a functional nasolacrimal blockage, may be more difficult to cure. We wanted to assess whether any simple, easily performed and minimally invasive procedure would help the symptoms of such patients without recourse to elaborate investigation or major surgery. A randomised study was performed to assess the effect of retropunctal cautery (RPC) and one-snip punctoplasty on the symptoms of patients with a presumed functional blockage.

\section{Patients and methods}

Patients attending the adnexal clinic of our hospital with symptoms of epiphora only were entered into the study after full information of their condition and procedures entailed was provided. A full history was obtained from each patient, followed by slit lamp examination. Patients were excluded if they had any of the following: a history suggestive of allergy, herpetic infection, trauma, facial palsy, previous nasal or lacrimal investigations/procedures, bleeding disorder, blepharitis, trichiasis, eyelid malposition, scarring or laxity, punctal abnormality, lacrimal fistula or mucocoele. A fluorescein dye disappearance test was then performed and, if positive, syringing of the nasolacrimal system was carried out. If the system was patent, randomisation was then undertaken for either no further procedure (group A), or minor surgery (group B).

Surgery comprised local infiltration of the medial aspect of the eyelid with lignocaine $1 \%$ with adrenaline (1:200 000) solution followed by a double horizontal line of retropunctal burns with a disposable monopolar cautery and a onesnip vertical punctoplasty using a fine spring scissor.

Patients in each group were asked to use chloramphenicol drops four times daily for 7 days. All patients were reviewed at 3 months to assess their symptoms.

\section{Results}

We performed 15 cases of syringing alone (group A) and 15 cases of syringing combined with RPC and a one-snip punctoplasty (group B). Sixty-three per cent of patients were female, and the average age was 69.2 years (range 30-89 years). Three months after treatment, six patients in group A were improved, compared with 13 in group B (Fisher's exact test, $p=0.0096)$.

\section{Discussion}

There is no doubt that obstruction of the nasolacrimal system can be successfully managed by surgery. ${ }^{2,3}$ However, the treatment of a functional or non-anatomical block is more inconsistent. Further investigation of this type of problem in the form of either a dacryocystogram or, ideally, lacrimal
S.A. Sadiq

R.N. Downes Department of Ophthalmology Queen's Medical Centre Nottingham NG7 2UH, UK 
scintillography is usually required, before resorting to surgical treatment. A recent survey has shown that only $41 \%$ of surgeons with a lacrimal interest would perform a dacryocystorhinostomy (DCR) for a lacrimal pump 'functional block'. It is for this reason of low surgical intervention that we wanted to investigate whether a simple acceptable technique (such as RPC and one-snip punctoplasty) may offer some improvement for these patients. Although our study involved younger patients also, most patients with a functional block tend to be older (the median age of the patients in our study was 73 years). It may thus also be for reasons of age that a major lacrimal procedure (which is usually performed under general anaesthesia) is avoided.

Although the follow-up is short at 3 months, we are confident that at least in the short term we are able to improve the symptoms of a substantial proportion of our patients with a functional nasolacrimal obstruction. This short-term relief is achieved by relatively simple methods that can be performed during the initial outpatient assessment. We are unsure of the exact mechanism whereby an RPC and one-snip punctoplasty actually improves tear outflow physiologically, but this combination of procedures may help by encouraging tears to more easily enter the punctum and canaliculi. The remainder of the lacrimal pump mechanism consists of canalicular shortening and lacrimal sac expansion on eyelid closure, followed by lacrimal sac collapsing and the forcing of tears into the nasolacrimal duct on eyelid opening. ${ }^{5}$ The RPC may cause tightening of the medial aspect of the eyelid to a mild degree, but punctal enlargement by itself would probably not result in an improvement, as patients in group A had also undergone punctal dilation (although not permanent) during the process of syringing. This improvement may be enough to improve quality of life without resort to major surgery, especially for older patients.

In conclusion, we would recommend that patients who suffer from a pump or functional nasolacrimal obstruction should receive RPC and a one-snip punctoplasty following the demonstration of a patent system on syringing. These simple procedures would also not impair further investigation or lacrimal surgery if required in the future.

\section{References}

1. Welham RAN. Lacrimal surgery. In: Collin JRO, editor. A manual of systematic eyelid surgery. 2nd ed. Edinburgh: Churchill Livingstone, 1989:109-20.

2. Tarbet KJ, Custer PL. External dacryocystorhinostomy: surgical success, patient satisfaction and economic cost. Ophthalmology 1995;102:1065-70.

3. Zolli CL, Shannon GM. Dacryocystorhinostomy: a review of 106 operations. Ophthalmic Surg 1982;13:905-10.

4. Conway ST, Wobig J. Evaluation and management of 'functional' nasolacrimal blockage: results of a survey of the American Society of Ophthalmic Plastic and Reconstructive Surgery. Ophthalmic Plastic Reconstr Surg 1994;10:185-8.

5. Jones LT, Wobig JL. Newer concepts of tear duct and eyelid anatomy and treatment. Trans Am Acad Ophthalmol Otolaryngol 1977;83:603-16. 